\title{
Analysis of Sturm-Liouville Eigenproblem with Interior Singularities and a Perturbation Parameter
}

\author{
Thomas Mbah Acho \\ Department of Mathematics and Applied Mathematics, University of the Free State, P.O. Box 339, Bloemfontein 9300, South Africa \\ Correspondence should be addressed to Thomas Mbah Acho; achotm@ufs.ac.za
}

Received 30 April 2014; Accepted 11 June 2014; Published 8 July 2014

Academic Editor: Abdon Atangana

Copyright (C) 2014 Thomas Mbah Acho. This is an open access article distributed under the Creative Commons Attribution License, which permits unrestricted use, distribution, and reproduction in any medium, provided the original work is properly cited.

\begin{abstract}
We devote this work to the discussion underpinning the derivation of eigenvalues and eigenfunction solutions for Sturm-Liouville boundary value problems. The study reveals that the parameter dependent nonstandard Sturm-Liouville boundary value problem with interior singularities may have more than two turning points. The Titchmarsh-Weyl $m$-function theory is applied here to obtain eigenfunction solutions valid for the whole interval in which pole singularities and two turning points are present. For the first time, with minimal constraints, the validity of the eigenfunction solutions are discussed when there are more than two turning points present. The eigenvalues are subsequently derived.
\end{abstract}

\section{Introduction}

The nonstandard Sturm-Liouville (SL) boundary value problems with interior singularities and transition points may require a refinement of some perturbation method or a completely new approach to enable us obtain eigenfunction solutions valid within the entire interval. There have been recent interest in the study of both the standard and nonstandard Sturm-Liouville boundary value problems and other similarly perturbed systems (see [1-9]). Cases where the interior singularities are simple poles or double poles with supplementary one or two turning points (transition points) have been extensively studied (see [10-16]). One method that has been shown to solve some of the SL eigenproblems with interior singularities is the Titchmarsh-Weyl $m$-function method $[10,11]$ (we refer the reader to whom the TitchmarshWeyl $m$-function theory is unfamiliar to [17]). However, there has not been any presentation where these interior singularities are associated to more than two turning points which arise naturally from a nonrestricted parameter. The discussion underpinning the turning point analysis will be presented in Section 2.2. The motivation of this study is based on the fact that a typical example of a nonstandard SturmLiouville equation with a nonrestricted parameter occurs naturally in the high energy resonance contributing equations, which in this case happens to be the corresponding radial
Schrödinger operator in $\mathbb{R}$. The fact that SL eigenproblems with interior singularities (which could either be a simple pole or a double pole singularity) could have a possibility of the presence of more than two turning points requires the introduction of a suitable constraint to enable the use of the Titchmarsh-Weyl $m$-function technique. We will now give a brief background below of the nature of this problem and in subsequent sections use this example to demonstrate how the Titchmarsh-Weyl $m$-function method can be applied to obtain eigenfunction solutions for a nonrestricted parameter dependent SL eigenproblem.

The resonance phenomenon appears in most material sciences and partly explains certain cases where structures such as bridges and buildings collapse (see [18]). Resonances are defined as poles of an analytic continuation of the quadratic form of the resolvent to the Riemann sheet through the branch cut along the continuous spectrum (see [19]). There have been studies ([20] and more recently [19]) on the investigation of resonances for the Hamiltonian operator. Asymptotic solutions to resonance equations of the Hamiltonian operator,

$$
H=-\left(\frac{1}{2}\right) \Delta-\frac{z}{r}+q(r),
$$

acting on $L^{2}\left(\mathbb{R}^{3}\right)$ for $z>0, r=|x|=\left(x_{1}^{2}+x_{2}^{2}+x_{3}^{2}\right)^{1 / 2}$, $x \in \mathbb{R}^{3}$, with appropriate conditions on a spherical symmetric 
support function $q(r)$, were presented in [19]. Eigenfunctions solutions for these operators are valid in the region $0<$ $r \leq b<\infty$. These solutions are singular at $r=0$. On considering the operator $H$ acting on $\mathbb{R}$ the desired eigenfunction solutions should satisfy boundary conditions for $r \in[a, b], a<0<b$ where $a$ and/or $b$ could be infinite. In this case the eigenfunctions solutions presented in [19] would not be valid for the entire interval because of the pole singularities at $r=0$ and the behaviour of these functions (which are the Whittaker functions) as $r \rightarrow \infty$. In this work we use the following condition on the perturbation term $q(r)$ :

$$
q(r)=\varepsilon \geq 0
$$

and we examine the case when

$$
q(r)=\varepsilon_{0}+\varepsilon r
$$

From [19], the radial Schrödinger equation then takes the form

$$
\left(-H_{l}+E\right) \Psi=\left(\frac{d^{2}}{d r^{2}}-\frac{l(l+1)}{r^{2}}+\frac{2 z}{r}-\varepsilon+\tau^{2}\right) \Psi=0,
$$

where $\tau$ is the spectral parameter introduced in [19], such that $E=\tau^{2}$ and the Riemann sheet corresponds to the lower half plane of $\tau$. With specification of $q(r)$ we can maintain the definition of resonances as in Theorem 1.2 of [19] as those points $E$ of the second Riemann sheet that can be represented in the form $E=\tau^{2}$, where $\tau$ belongs to the lower half plane (that is $\operatorname{Im} \tau<0$ ). It may be worth mentioning that even in a more simple case like the vibration of a circular membrane [18], the state of resonance is determined by the resulting radial equation. The objective of this work is not to solve the resonance equations as in [19] but to obtain eigenfunctions solutions and eigenvalues valid for the entire interval. We find that (4) has Whittaker functions as the eigenfunction solutions for $r \in \mathbb{R}^{3}$ as well as for $r \in \mathbb{R}$, where $r$ is finite and different from zero. However, the case for $r \in \mathbb{R}$, with boundary conditions such that $r \in[a, b]$ where $a \leq 0 \leq b$, presents difficulties in obtaining the eigenfunctions solutions as highlighted above. In Section 3 we present these solutions and in Section 4 we derive the eigenvalue relations. The eigenfunction solutions will of course satisfy (4) for $r \in \mathbb{R}^{3}$ and the restricted interval $0<r \leq b<\infty$.

\section{Preliminaries and Turning Point Analysis}

2.1. Preliminaries with a Restricted Perturbation Term $q(r)=\varepsilon \geq 0$. Sequel to our discussion in the previous section we can rewrite (4) with the associated conditions as the following boundary value problem:

$$
\begin{gathered}
L \Psi=\left(\frac{d^{2}}{d r^{2}}+\frac{\left(\tau^{2}-\varepsilon\right) r^{2}+2 z r-l}{r^{2}}\right) \Psi=0 \\
\Psi(a)=\Psi(b)=0, \quad r \in[a, b] \subset \mathbb{R}, a<0<b,
\end{gathered}
$$

where $l=0,1, \ldots ; z>0, \varepsilon>0$ and $\operatorname{Im} \tau<0$.
The differential equation (5) has the following turning points:

$$
\rho_{1,2}=\frac{-z+\left[z^{2}+l(l+1)\left(\tau^{2}-\varepsilon\right)\right]^{1 / 2}}{\tau^{2}-\varepsilon} .
$$

The value of the angular momentum $l$ is different from zero and $\left(\tau^{2}-\varepsilon\right)$ is complex; the turning points $\rho_{1}$ and $\rho_{2}$ are distinct (i.e., do not coincide). Therefore, the eigenfunctions solutions of (5) will be in terms of some asymptotic approximation of Whittaker functions (see [21-24]). Using the transformation

$$
x=2 i\left(\tau^{2}-\varepsilon\right)^{1 / 2} r, \quad \Psi(r)=\Phi(x),
$$

equation (5) becomes

$$
L \Phi=\left(\frac{d^{2}}{d x^{2}}-\left[\frac{1}{4}+\frac{i z\left(\tau^{2}-\varepsilon\right)^{-1 / 2}}{x}+\frac{l(l+1)}{x^{2}}\right]\right) \Phi=0 .
$$

Consider

$$
L \Phi=\left(\frac{d^{2}}{d x^{2}}+\left[-\frac{1}{4}+\frac{k}{x}+\frac{1 / 4-\mu^{2}}{x^{2}}\right]\right) \Phi=0,
$$

whose eigenfunctions solution in terms of the $M(\cdot)$ and $W(\cdot)$ Whittaker functions is

$$
\Phi(x)=\alpha M_{k, \mu}(x)+\beta W_{k, \mu}(x),
$$

where $\alpha$ and $\beta$ are constants that would be determined. On comparison of (9) and (10), we obtain

$$
k=-i z\left(\tau^{2}-\varepsilon\right)^{-1 / 2}, \quad \mu=l+\frac{1}{2} .
$$

From whence we may then write the solution of (5) to be of the form

$$
\Psi(r)=\alpha M_{k, \mu}\left(2 i\left[\tau^{2}-\varepsilon\right]^{1 / 2} r\right)+\beta W_{k, \mu}\left(2 i\left[\tau^{2}-\varepsilon\right]^{1 / 2} r\right),
$$

where $k$ and $\mu$ are as in (12). It is obvious that for $0<r \leq b<$ $\infty$ and $\varepsilon=0 ; M_{k, \mu}(\cdot)$ and $W_{k, \mu}(\cdot)$ as presented in (13) give the two linearly independent solutions of the unperturbed equation as in [19]. However, considering an interval $[a, b]$ that includes the double pole singularity at $r=0$ and the possibility that $a$ and/or $b$ could be infinite results in the fact that for $\left|\tau^{2}-\varepsilon\right|$ small, the internal boundary layer in which the asymptotic series for $M_{k, \mu}(\cdot)$ and $W_{k, \mu}(\cdot)$ are inaccurate includes the whole interval $[a, b]$. This is as a result of the prominence in the effect of the double pole for $\left|\tau^{2}-\varepsilon\right|$ small and the presence of the turning points that lie in the complex plane. Some of the behavioural properties of the $M_{k, \mu}(\cdot)$ and $W_{k, \mu}(\cdot)$ Whittaker functions are presented in $[10,25]$.

The method of solution we employ requires that we determine the quadrant in the complex plane on which 


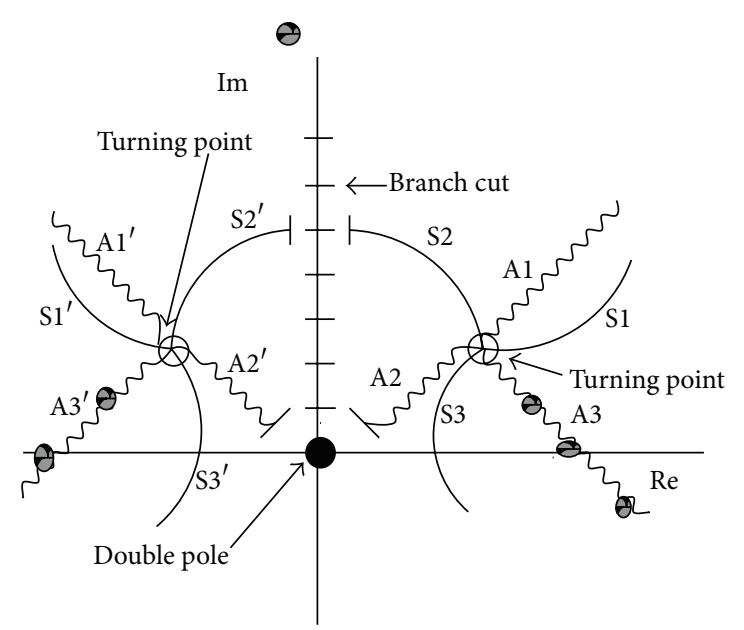

Figure 1: Turning points both lie in the upper half of the complex plane and two of the zeros of the eigenfunctions lie on the real axis. In Figures 1-4, $A j$ and $A j^{\prime}$ and $j=1,2,3$ are antistokes lines; $S j$ and $S j^{\prime}$ and $j=1,2,3$ are stokes lines and zeros of eigenfunctions.

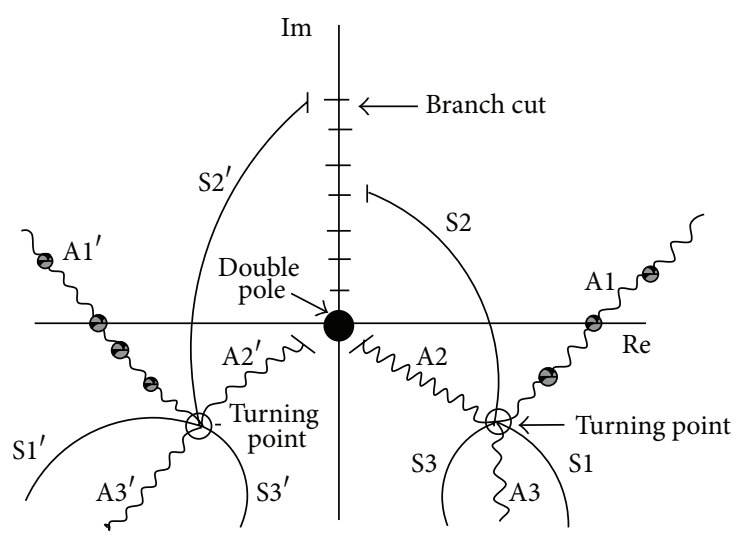

FIGURE 2: Turning points both lie in the lower half plane and two of the zeros of the eigenfunctions lie on the real axis.

the turning points lie. Considering the resonance condition $\operatorname{Im} \tau<0$, which implies that either

$$
\left(\operatorname{Im} \tau^{2}>0, \operatorname{Re} \tau<0\right) \quad \text { or } \quad\left(\operatorname{Im} \tau^{2}<0, \operatorname{Re} \tau>0\right)
$$

and the fact that $\tau^{2}$ appears in both the discriminant and the denominator of (7) means that the turning points could lie in any of the four quadrants. Our method is applicable with the choice of branch cuts as in the theory of atmospheric and ocean waves. The locations of the turning points on the complex plane are as in the Figures 1,2,3, and 4. These figures also indicate the stokes lines and antistokes lines and how one of the zeros of the Whitaker function does lie on the positive real axis and the other one on the negative real axis which is essential for the boundary conditions to be satisfied.

Our method would require the use of Titchmarsh-Weyl $m$-function $[10,17]$, so we shall only be interested in the turning points that are separated such that one lies in the left half of the complex plane and the other in the right half. The Titchmarsh-Weyl $m$-function theory for (5) with two singular points $a, b$ requires that we choose a regular point $c \in[a, b]$. We then let

$$
\Psi(r, \widehat{\tau} ; k, \mu)=\Theta(r, \widehat{\tau} ; k, \mu) \Phi(r, \widehat{\tau} ; k, \mu)
$$

Denote the fundamental solution of (5), defined by

$$
\left[\begin{array}{cc}
\Theta(c, \widehat{\tau} ; k, \mu) & \Phi(c, \widehat{\tau} ; k, \mu) \\
\Theta^{\prime}(c, \widehat{\tau} ; k, \mu) & \Phi^{\prime}(c, \widehat{\tau} ; k, \mu)
\end{array}\right]=\left(\begin{array}{ll}
1 & 0 \\
0 & 1
\end{array}\right),
$$

where in this case $\widehat{\tau}=\left(\tau^{2}-\varepsilon\right)$. The Titchmarsh-Weyl $m$ function at $r=a$ and $r=b$ is defined, respectively, by

$$
\begin{aligned}
& m^{-}(\widehat{\tau})=\lim _{r \rightarrow a} \frac{\Theta(r, \widehat{\tau} ; k, \mu)}{\Phi(r, \widehat{\tau} ; k, \mu)}, \\
& m^{+}(\widehat{\tau})=-\lim _{r \rightarrow b} \frac{\Theta(r, \widehat{\tau} ; k, \mu)}{\Phi(r, \widehat{\tau} ; k, \mu)}
\end{aligned}
$$




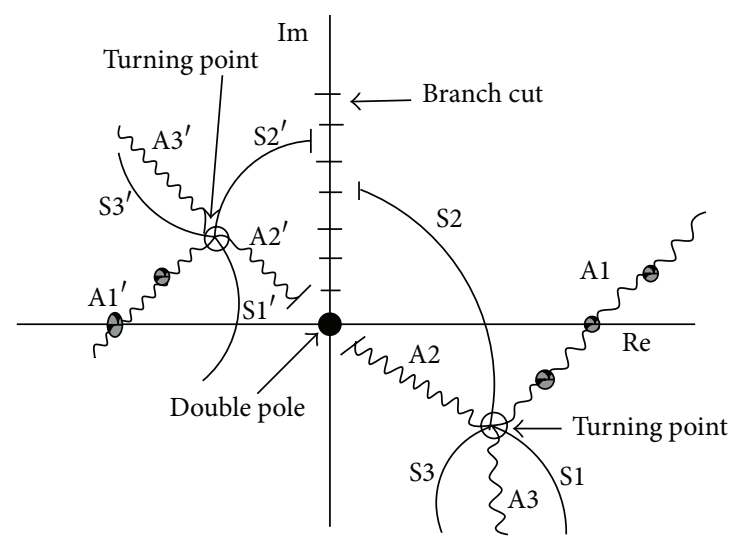

FIGURE 3: One of the turning points lie in the lower half of the complex plane and the other one in the upper half plane.

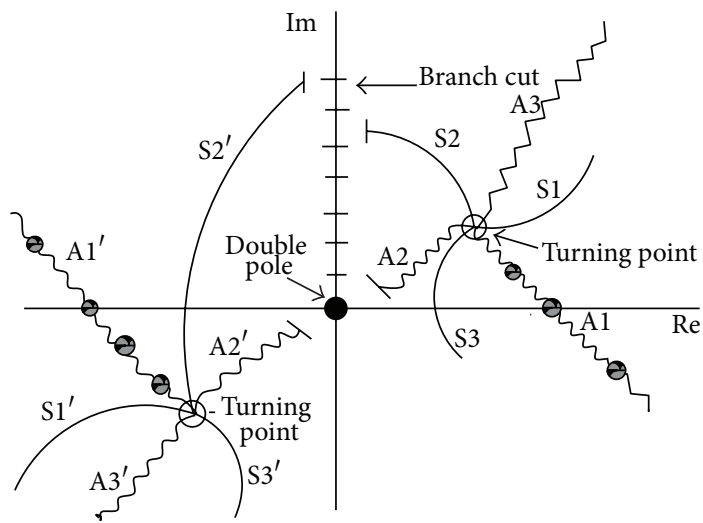

FIGURE 4: One of the turning points lie in the lower half of the complex plane and the other one in the upper half plane.

for $\operatorname{Im} \tau \neq 0$. Both $m^{-}(\widehat{\tau})$ and $m^{+}(\widehat{\tau})$ are analytic functions in both $\operatorname{Im}(\widehat{\tau})>0$ and $\operatorname{Im}(\widehat{\tau})<0$ and obey the following relations:

$$
\begin{array}{ll}
m^{-}(\overline{\widehat{\tau}})=\overline{m^{-}(\widehat{\tau})}, & \operatorname{Im} \widehat{\tau} \operatorname{Im} m^{-}(\widehat{\tau})<0, \\
m^{+}(\overline{\widehat{\tau}})=\overline{m^{+}(\widehat{\tau})}, \quad \operatorname{Im} \widehat{\tau} \operatorname{Im} m^{+}(\widehat{\tau})>0 .
\end{array}
$$

Further, if we define $\Psi^{ \pm}(r, \widehat{\tau} ; k, \mu)$ by

$$
\Psi^{ \pm}(r, \widehat{\tau} ; k, \mu)=\Theta(r, \widehat{\tau} ; k, \mu)+m^{ \pm} \Phi(r, \widehat{\tau} ; k, \mu)
$$

then it follows that

$$
\Psi^{+}(r, \widehat{\tau} ; k, \mu) \in L^{2}([c, b]), \quad \Psi^{-}(r, \widehat{\tau} ; k, \mu) \in L^{2}([a, c]) .
$$

We use the above to construct our solutions in Section 3. Before doing so we present in the proceeding subsection a turning point analysis for the nonrestricted perturbation parameter.

\subsection{Turning Point Analysis for the Nonrestricted Perturbation} Parameter $\varepsilon(r)=\varepsilon_{0}+\varepsilon_{1} r$. The turning point analysis for $\varepsilon(r)=\varepsilon_{0}=\varepsilon$ (notation used in the preceding and proceeding sections) would be similar to that presented in [10], so for the sake of brevity it would not be presented here. The corresponding Schrödinger equation for the spectral parameter $k$ and the perturbation parameter $\varepsilon(r)=\varepsilon_{0}+\varepsilon_{1} r$, $\varepsilon_{1} \neq 0$ is given by

$$
L \Psi=\left(\frac{d^{2}}{d r^{2}}-\frac{l(l+1)}{r^{2}}-\left(\varepsilon_{0}+\varepsilon_{1} r\right)+k^{2}\right) \Psi=0 .
$$

We rewrite this in the form

$$
\left(\frac{d^{2}}{d r^{2}}-\frac{\varepsilon_{1} r^{3}-\left(k^{2}-\varepsilon_{0}\right) r^{2}+l(l+1)}{r^{2}}\right) \Psi=0 .
$$

For $r \in \mathbb{R}$, we may consider the generalized interval $r \in(a, b), a<0<b$, with no restrictions on $a$ and $b$. Furthermore, for the purpose of proper analysis of the turning points we will choose the boundary conditions as

$$
\Psi(a)=\Psi(b)=0 .
$$

The boundary value problems (22) and (23) have a double pole singularity, at $r=0$, and three turning points which we now determine. The turning points are located at $r=\eta$ for which

$$
r^{3}-\frac{\left(k^{2}-\varepsilon_{0}\right) r^{2}}{\varepsilon_{1}}+\frac{l(l+1)}{\varepsilon_{1}}=0 .
$$


We make the substitution

$$
r=y+\frac{\left(k^{2}-\varepsilon_{0}\right)}{3 \varepsilon_{1}}
$$

to reduce (24) to the depressed cubic

$$
y^{3}+\lambda y+\xi=0
$$

where $\lambda=-(1 / 3)\left(\left(k^{2}-\varepsilon_{0}\right) / \varepsilon_{1}\right)^{2}$ and $\xi=(2 / 27)\left(\left(k^{2}-\varepsilon_{0}\right) /\right.$ $\left.\varepsilon_{1}\right)^{2}+l(l+1) / \varepsilon_{1}$.

One of the roots of the depressed cubic is

$$
y_{1}=\sqrt[3]{\frac{-\xi}{2}+\sqrt{\frac{\xi^{2}}{4}+\frac{\lambda^{3}}{27}}}+\sqrt[3]{\frac{-\xi}{2}-\sqrt{\frac{\xi^{2}}{4}+\frac{\lambda^{3}}{27}}} .
$$

We then obtain the remaining roots as

$$
y_{2,3}=\frac{-y_{1}+\left(-4 \lambda-3 y_{1}^{2}\right)^{1 / 2}}{2} .
$$

From (24) to (28) the three turning points are as follows:

$$
\begin{gathered}
\eta_{1}=y_{1}+\frac{k^{2}-\varepsilon_{0}}{3 \varepsilon_{1}}, \\
\eta_{2,3}=\frac{-y_{1}+\left(-4 \lambda-3 y_{1}^{2}\right)^{1 / 2}}{2}+\frac{k^{2}-\varepsilon_{0}}{3 \varepsilon_{1}} .
\end{gathered}
$$

The turning points coalesce into a single turning point when $y_{1}=(-2 \lambda / 3)^{1 / 2}$ and there are two distinct turning points if $y_{1}=(-4 \lambda / 3)^{1 / 2}$. For any other values of $y_{1}$ there will be three distinct turning points located on the complex plane.

In order to apply the Titchmarsh-Weyl $m$-function technique for reasons stated in Section 2.1, there should be two distinct turning points; therefore, we must have the restriction that

$$
y_{1}=\left(-\frac{4 \lambda}{3}\right)^{1 / 2}=\frac{2}{3}\left(\frac{k^{2}-\varepsilon_{0}}{\varepsilon_{1}}\right) .
$$

This provides the split intervals required for the application of the Titchmarsh-Weyl $m$-function theory which will be similar to that when $\varepsilon=\varepsilon_{0}, \varepsilon_{1}=0$; provided of course that one turning point lies in the left half and the other in the right half of the complex plane.

\section{Eigenfunction Solutions}

The analysis in Section 2 is essential for the application of the method of solutions we now provide here and in the section that follows. We will assume that the turning point $\rho_{2}$ lies in the left half plane and $\rho_{1}$ lies in the right half plane. We split the interval $[a, b]$ as follows:

$$
\begin{array}{ll}
I_{1}=\left[a, \rho_{2}^{*}\right], & I_{2}=\left[\rho_{2}^{*}, 0\right], \\
I_{3}=\left[0, \rho_{1}^{*}\right], & I_{4}=\left[\rho_{1}^{*}, b\right],
\end{array}
$$

where $\rho_{j}^{*}=\operatorname{Re} \rho_{j}, j=1,2$.
We obtain our solution for (5) as a linear combination of eigenfunctions for the split intervals and it turns out that the boundary conditions (6) are not necessary as they are automatically satisfied by our solution. The following theorem summarizes our first results.

Theorem 1. Let $\Theta(r, \widehat{\tau} ; k, \mu)$ and $\Phi(r, \widehat{\tau} ; k, \mu)$ be appropriate combinations of the eigenfunctions solutions of (5) with (6) for $|\widehat{\tau}|=\left|\tau^{2}-\varepsilon\right|$ small such that

$$
\begin{aligned}
& m^{+}(\widehat{\tau})=-\lim _{r \rightarrow b} \frac{\Theta(r, \widehat{\tau} ; k, \mu)}{\Phi(r, \widehat{\tau} ; k, \mu)}, \\
& m^{-}(\widehat{\tau})=-\lim _{r \rightarrow 0} \frac{\Theta(r, \widehat{\tau} ; k, \mu)}{\Phi(r, \widehat{\tau} ; k, \mu)}, \\
& n^{+}(\widehat{\tau})=-\lim _{r \rightarrow 0} \frac{\Theta(r, \widehat{\tau} ; k, \mu)}{\Phi(r, \widehat{\tau} ; k, \mu)}, \\
& n^{-}(\widehat{\tau})=-\lim _{r \rightarrow a} \frac{\Theta(r, \widehat{\tau} ; k, \mu)}{\Phi(r, \widehat{\tau} ; k, \mu)} .
\end{aligned}
$$

Then the solutions to (5) would be

$$
\begin{aligned}
\Psi(r, \widehat{\tau} ; k, \mu)=\Theta(r, \widehat{\tau} ; k, \mu) \\
+\left\{\begin{array}{l}
m^{+}(\widehat{\tau}) \Phi(r, \widehat{\tau} ; k, \mu) \in L^{2}\left(\left[\rho_{1}^{*}, b\right]\right) \\
m^{-}(\widehat{\tau}) \Phi(r, \widehat{\tau} ; k, \mu) \in L^{2}\left(\left[0, \rho_{1}^{*}\right]\right) \\
n^{+}(\widehat{\tau}) \Phi(r, \widehat{\tau} ; k, \mu) \in L^{2}\left(\left[\rho_{2}^{*}, 0\right]\right) \\
n^{-}(\widehat{\tau}) \Phi(r, \widehat{\tau} ; k, \mu) \in L^{2}\left(\left[a, \rho_{2}^{*}\right]\right),
\end{array}\right.
\end{aligned}
$$

where $m^{+}, m^{-}, n^{+}, n^{-}$are as defined in Section 2.1.

Proof. For convenience we suppress the $k, \mu$ dependence of $\Theta$ and $\Phi$. We start with the regular solutions $M_{k, \mu}\left(2 i \widehat{\tau}^{1 / 2} r\right)$ and $W_{k, \mu}\left(2 i \widehat{\tau}^{1 / 2} r\right)$ of (5) on $I_{3} \cup I_{4}$, where $\hat{t}=\left(\tau^{2}-\varepsilon\right), k=$ $i z\left(\tau^{2}-\varepsilon\right)^{1 / 2}$, and $\mu=l+1 / 2$.

Thus we let

$$
\begin{aligned}
& \Theta(r, \widehat{\tau})=\alpha_{0}^{+} M_{k, \mu}\left(2 i \widehat{\tau}^{1 / 2} r\right)+\alpha_{1}^{+} M_{k,-\mu}\left(2 i \widehat{\tau}^{1 / 2} r\right), \\
& \Phi(r, \widehat{\tau})=\beta_{0}^{+} W_{k, \mu}\left(2 i \hat{\tau}^{1 / 2} r\right)+\beta_{1}^{+} W_{-k, \mu}\left(2 i \hat{\tau}^{1 / 2} r\right) .
\end{aligned}
$$

We now proceed to determine $\alpha_{0}^{+}, \alpha_{1}^{+}, \beta_{0}^{+}$, and $\beta_{1}^{+}$. Substituting (34) into (16) we obtain

$$
\begin{aligned}
& \Theta\left(\rho_{1}^{*}, \widehat{\tau}\right)=\alpha_{0}^{+} M_{k, \mu}\left(2 i \hat{\tau}^{1 / 2} \rho_{1}^{*}\right)+\alpha_{1}^{+} M_{k,-\mu}\left(2 i \hat{\tau}^{1 / 2} \rho_{1}^{*}\right)=1, \\
& \Theta^{\prime}\left(\rho_{1}^{*}, \widehat{\tau}\right) \\
& =\alpha_{0}^{+}\left\{\frac{\left(1+2 \mu-4 i \widehat{\tau}^{1 / 2} \rho_{1}^{*}\right)}{2 i \hat{\tau}^{1 / 2} \rho_{1}^{*}} M_{k, \mu}\left(2 i \hat{\tau}^{1 / 2} \rho_{1}^{*}\right)\right. \\
& \left.\quad+\frac{(2 \mu-2 k+1)}{(2 \mu+1)\left(2 i \widehat{\tau}^{1 / 2} \rho_{1}^{*}\right)} M_{k-1 / 2, \mu+1 / 2}\left(2 i \widehat{\tau}^{1 / 2} \rho_{1}^{*}\right)\right\}
\end{aligned}
$$




$$
\begin{aligned}
+\alpha_{1}^{+}\left\{\frac{\left(1-2 \mu-4 i \hat{\tau}^{1 / 2} \rho_{1}^{*}\right)}{4 i \hat{\tau}^{1 / 2} \rho_{1}^{*}} M_{k,-\mu}\left(2 i \bar{\tau}^{1 / 2} \rho_{1}^{*}\right)\right. & \beta_{1}^{+}=\frac{-1}{Q_{2}}\left\{\frac{\left(1+2 \mu-4 i \bar{\tau}^{1 / 2} \rho_{1}^{*}\right)}{2 i \hat{\tau}^{1 / 2} \rho_{1}^{*}} W_{k, \mu}\left(2 i \hat{\tau}^{1 / 2} \rho_{1}^{*}\right)\right. \\
\left.+\frac{(1-2 \mu-2 k)}{(1-2 \mu)\left(2 i \hat{\tau}^{1 / 2} \rho_{1}^{*}\right)^{1 / 2}} M_{k-1 / 2, \mu+1 / 2}\left(2 i \hat{\tau}^{1 / 2} \rho_{1}^{*}\right)\right\} & \left.+\frac{(2 k-2 \mu-1)}{\left(2 i \hat{\tau}^{1 / 2} \rho_{1}^{*}\right)^{1 / 2}} W_{k-1 / 2, \mu+1 / 2}\left(-2 i \hat{\tau}^{1 / 2} \rho_{1}^{*}\right)\right\},
\end{aligned}
$$

From (36) we obtain the following:

where

$\alpha_{0}^{+}$

$$
\begin{aligned}
=\frac{1}{Q_{1}}\{ & \frac{\left(1-2 \mu-4 i \hat{\tau}^{1 / 2} \rho_{1}^{*}\right)}{4 i \widehat{\tau}^{1 / 2} \rho_{1}^{*}} M_{k, \mu}\left(2 i \widehat{\tau}^{1 / 2} \rho_{1}^{*}\right) \\
& \left.+\frac{(1-2 \mu-2 k)}{(1-2 \mu)\left(2 i \hat{\tau}^{1 / 2} \rho_{1}^{*}\right)^{1 / 2}} M_{k-1 / 2,-\mu+1 / 2}\left(2 i \hat{\tau}^{1 / 2} \rho_{1}^{*}\right)\right\},
\end{aligned}
$$

$\alpha_{1}^{+}$

$$
\begin{aligned}
=\frac{-1}{Q_{1}}\{ & \frac{\left(1+2 \mu-4 i \hat{\tau}^{1 / 2} \rho_{1}^{*}\right)}{2 i \hat{\tau}^{1 / 2} \rho_{1}^{*}} M_{k, \mu}\left(2 i \hat{\tau}^{1 / 2} \rho_{1}^{*}\right) \\
& \left.+\frac{(2 \mu-2 k+1)}{(2 \mu+1)\left(2 i \hat{\tau}^{1 / 2} \rho_{1}^{*}\right)^{1 / 2}} M_{k-1 / 2, \mu+1 / 2}\left(2 i \hat{\tau}^{1 / 2} \rho_{1}^{*}\right)\right\},
\end{aligned}
$$

where

$Q_{1}$

$$
\begin{aligned}
= & M_{k, \mu}\left(2 i \hat{\tau}^{1 / 2} \rho_{1}^{*}\right) \\
& \times\left\{\frac{\left(1-2 \mu-4 i \hat{\tau}^{1 / 2} \rho_{1}^{*}\right)}{4 i \hat{\tau}^{1 / 2} \rho_{1}^{*}} M_{k,-\mu}\left(2 i \hat{\tau}^{1 / 2} \rho_{1}^{*}\right)\right. \\
& \left.\quad+\frac{(1-2 \mu-2 k)}{(1-2 \mu)\left(2 i \hat{\tau}^{1 / 2} \rho_{1}^{*}\right)^{1 / 2}} M_{k-1 / 2,-\mu-1 / 2}\left(2 i \widehat{\tau}^{1 / 2} \rho_{1}^{*}\right)\right\} \\
+ & \left(-M_{k,-\mu}\left(2 i \bar{\tau}^{1 / 2} \rho_{1}^{*}\right)\right. \\
& \times\left\{\frac{\left(1+2 \mu-4 i \hat{\tau}^{1 / 2} \rho_{1}^{*}\right)}{2 i \hat{\tau}^{1 / 2} \rho_{1}^{*}} M_{k, \mu}\left(2 i i^{1 / 2} \rho_{1}^{*}\right)\right. \\
& \left.\left.+\frac{(2 \mu-2 k+1)}{(2 \mu+1)\left(2 i \hat{\tau}^{1 / 2} \rho_{1}^{*}\right)^{1 / 2}} M_{k-1 / 2, \mu+1 / 2}\left(2 i \hat{\tau}^{1 / 2} \rho_{1}^{*}\right)\right\}\right) .
\end{aligned}
$$

Similarly, substitution of (35) into (16) yields

$$
\begin{aligned}
\beta_{0}^{+}=\frac{1}{Q_{2}}\left\{\frac{\left(1+2 \mu+4 i \hat{\tau}^{1 / 2} \rho_{1}^{*}\right)}{4 i \hat{\tau}^{1 / 2} \rho_{1}^{*}} W_{-k, \mu}\left(-2 i \hat{\tau}^{1 / 2} \rho_{1}^{*}\right)\right. \\
\left.+\left(-\frac{(1+2 \mu+2 k)}{\left(-2 i \hat{\tau}^{1 / 2} \rho_{1}^{*}\right)^{1 / 2}} W_{-k+1 / 2, \mu+1 / 2}\left(-2 i \hat{\tau}^{1 / 2} \rho_{1}^{*}\right)\right)\right\},
\end{aligned}
$$

$$
\begin{aligned}
& Q_{2} W_{k, \mu}\left(2 i \widehat{\tau}^{1 / 2} \rho_{1}^{*}\right) \\
& \quad \times\left\{\frac{\left(1+2 \mu+4 i \hat{\tau}^{1 / 2} \rho_{1}^{*}\right)}{4 i \hat{\tau}^{1 / 2} \rho_{1}^{*}} W_{-k, \mu}\left(-2 i \hat{\tau}^{1 / 2} \rho_{1}^{*}\right)\right. \\
& \left.+\left(-\frac{(1+2 \mu+2 k)}{\left(-2 i \widehat{\tau}^{1 / 2} \rho_{1}^{*}\right)^{1 / 2}} W_{-k+1 / 2, \mu+1 / 2}\left(-2 i \widehat{\tau}^{1 / 2} \rho_{1}^{*}\right)\right)\right\} \\
& +\left(-W_{-k, \mu}\left(-2 i \widehat{\tau}^{1 / 2} \rho_{1}^{*}\right)\right. \\
& \times\left\{\frac{\left(1+2 \mu-4 i \hat{\tau}^{1 / 2} \rho_{1}^{*}\right)}{2 i \hat{\tau}^{1 / 2} \rho_{1}^{*}} W_{k, \mu}\left(2 i \widehat{\tau}^{1 / 2} \rho_{1}^{*}\right)\right. \\
& \left.\left.+\frac{(2 k-2 \mu-1)}{\left(2 i \widehat{\tau}^{1 / 2} \rho_{1}^{*}\right)^{1 / 2}} W_{k-1 / 2, \mu+1 / 2}\left(2 i \hat{\tau}^{1 / 2} \rho_{1}^{*}\right)\right\}\right) .
\end{aligned}
$$

Using (17) we may then find $m^{+}(\widehat{\tau})$ and $m^{-}(\widehat{\tau})$, respectively, at $r=b$ and $r=0$ as follows:

$$
m^{+}(\widehat{\tau})=-\lim _{r \rightarrow b} \frac{\Theta(r, \widehat{\tau})}{\Phi(r, \widehat{\tau})}, \quad m^{-}(\widehat{\tau})=-\lim _{r \rightarrow 0} \frac{\Theta(r, \widehat{\tau})}{\Phi(r, \widehat{\tau})},
$$

where $\Theta$ and $\Phi$ are as given in (34) and (35) with $\alpha_{j}, \beta_{j}, j=$ 0,1 as obtained in (37) to (40). From (19) we then have

$$
\begin{aligned}
& \Psi_{+}^{+}(r, \widehat{\tau}) \equiv \Theta(r, \widehat{\tau})+m^{+}(\widehat{\tau}) \Phi(r, \widehat{\tau}) \in L^{2}\left(\left[\rho_{1}^{*}, b\right]\right), \\
& \Psi_{+}^{-}(r, \widehat{\tau}) \equiv \Theta(r, \widehat{\tau})+m^{-}(\widehat{\tau}) \Phi(r, \widehat{\tau}) \in L^{2}\left(\left[0, \rho_{1}^{*}\right]\right) .
\end{aligned}
$$

Therefore, the eigenfunctions for $I_{3} \cup I_{4}$ are

$$
\Psi_{+}(r, \widehat{\tau})= \begin{cases}\Psi_{+}^{+}(r, \widehat{\tau}), & r \in\left[\rho_{1}^{*}, b\right] \\ \Psi_{+}^{-}(r, \widehat{\tau}), & r \in\left[0, \rho_{1}^{*}\right]\end{cases}
$$

which automatically satisfy the boundary conditions at $b$.

Now, for the interval $I_{1} \cup I_{2}$, we let

$$
\begin{aligned}
& \Theta(r, \widehat{\tau})=\alpha_{0}^{-} M_{k, \mu}\left(2 i \hat{\tau}^{1 / 2} r\right)+\alpha_{1}^{-} M_{k,-\mu}\left(2 i \hat{\tau}^{1 / 2} r\right), \\
& \Phi(r, \widehat{\tau})=\beta_{0}^{-} W_{k, \mu}\left(2 i \hat{\tau}^{1 / 2} r\right)+\beta_{1}^{-} W_{-k, \mu}\left(2 i{ }^{1 / 2} r\right) .
\end{aligned}
$$


Proceeding as above we obtain

$$
\begin{gathered}
\alpha_{0}^{-}=\frac{1}{Q_{3}}\left\{\frac{\left(1-2 \mu-4 i \widehat{\tau}^{1 / 2} \rho_{2}^{*}\right)}{4 i \widehat{\tau}^{1 / 2} \rho_{2}^{*}} M_{k, \mu}\left(2 i \widehat{\tau}^{1 / 2} \rho_{2}^{*}\right)\right. \\
\left.\quad+\frac{(1-2 \mu-2 k)}{(1-2 \mu)\left(2 i \hat{\tau}^{1 / 2} \rho_{2}^{*}\right)} M_{k-1 / 2,-\mu+1 / 2}\left(2 i \widehat{\tau}^{1 / 2} \rho_{2}^{*}\right)\right\}, \\
\alpha_{1}^{-} \\
=\frac{1}{Q_{3}}\left\{\frac{\left(1+2 \mu-4 i \widehat{\tau}^{1 / 2} \rho_{2}^{*}\right)}{2 i \hat{\tau}^{1 / 2} \rho_{2}^{*}} M_{k, \mu}\left(2 i \bar{\tau}^{1 / 2} \rho_{2}^{*}\right)\right. \\
\quad+\frac{(2 \mu-2 k+1)}{(1+2 \mu)\left(2 i \hat{\tau}^{1 / 2} \rho_{2}^{*}\right)^{1 / 2}} \\
\left.\quad \times M_{k-1 / 2, \mu+1 / 2}\left(2 i \widehat{\tau}^{1 / 2} \rho_{2}^{*}\right)\right\},
\end{gathered}
$$

where

$Q_{3}$

$$
\begin{aligned}
= & M_{k, \mu}\left(2 i \hat{\tau}^{1 / 2} \rho_{2}^{*}\right) \\
& \times\left\{\frac{\left(1-2 \mu-4 i \hat{\tau}^{1 / 2} \rho_{2}^{*}\right)}{4 i \widehat{\tau}^{1 / 2} \rho_{2}^{*}} M_{k,-\mu}\left(2 i \hat{\tau}^{1 / 2} \rho_{2}^{*}\right)\right. \\
& \left.+\frac{(1-2 \mu-2 k)}{(1-2 \mu)\left(2 i \widehat{\tau}^{1 / 2} \rho_{2}^{*}\right)^{1 / 2}} M_{k-1 / 2, \mu-1 / 2}\left(2 i \hat{\tau}^{1 / 2} \rho_{2}^{*}\right)\right\} \\
+ & \left(-M_{k,-\mu}\left(2 i \hat{\tau}^{1 / 2} \rho_{2}^{*}\right)\right. \\
\times & \frac{\left(1+2 \mu-4 i \hat{\tau}^{1 / 2} \rho_{2}^{*}\right)}{2 i \hat{\tau}^{1 / 2} \rho_{2}^{*}} M_{k, \mu}\left(2 i \widehat{\tau}^{1 / 2} \rho_{2}^{*}\right) \\
& \left.\left.+\frac{(2 \mu-2 k+1)}{(1+2 \mu)\left(2 i \widehat{\tau}^{1 / 2} \rho_{2}^{*}\right)^{1 / 2}} M_{k-1 / 2, \mu+1 / 2}\left(2 i \widehat{\tau}^{1 / 2} \rho_{2}^{*}\right)\right\}\right) .
\end{aligned}
$$

Using the same process we find that

$$
\begin{aligned}
& \beta_{0}^{-}=\frac{1}{Q_{4}}\left\{\frac{\left(1+2 \mu+4 i \widehat{\tau}^{1 / 2} \rho_{2}^{*}\right)}{4 i \widehat{\tau}^{1 / 2} \rho_{2}^{*}} W_{-k, \mu}\left(-2 i \widehat{\tau}^{1 / 2} \rho_{2}^{*}\right)\right. \\
& \left.+\left(-\frac{(1+2 \mu+2 k)}{\left(-2 i \widehat{\tau}^{1 / 2} \rho_{2}^{*}\right)^{1 / 2}} W_{-k+1 / 2, \mu+1 / 2}\left(-2 i \widehat{\tau}^{1 / 2} \rho_{2}^{*}\right)\right)\right\}, \\
& \beta_{1}^{-}=\frac{-1}{Q_{4}}\left\{\frac{\left(1+2 \mu-4 i \widehat{\tau}^{1 / 2} \rho_{2}^{*}\right)}{2 i \widehat{\tau}^{1 / 2} \rho_{2}^{*}} W_{k, \mu}\left(2 i \widehat{\tau}^{1 / 2} \rho_{2}^{*}\right)\right. \\
& \left.+\frac{(2 k-2 \mu-1)}{\left(2 i \widehat{\tau}^{1 / 2} \rho_{2}^{*}\right)^{1 / 2}} W_{k-1 / 2, \mu+1 / 2}\left(-2 i \widehat{\tau}^{1 / 2} \rho_{2}^{*}\right)\right\},
\end{aligned}
$$

where

$$
\begin{aligned}
& Q_{4} \\
& =W_{k, \mu}\left(2 i \widehat{\tau}^{1 / 2} \rho_{2}^{*}\right) \\
& \times\left\{\frac{\left(1+2 \mu+4 i \widehat{\tau}^{1 / 2} \rho_{2}^{*}\right)}{4 i \widehat{\tau}^{1 / 2} \rho_{2}^{*}} W_{-k, \mu}\left(-2 i \widehat{\tau}^{1 / 2} \rho_{2}^{*}\right)+\right. \\
& \left.+\left(-\frac{(1+2 \mu+2 k)}{\left(-2 i \widehat{\tau}^{1 / 2} \rho_{2}^{*}\right)^{1 / 2}} W_{-k+1 / 2, \mu+1 / 2}\left(-2 i \widehat{\tau}^{1 / 2} \rho_{2}^{*}\right)\right)\right\} \\
& +\left(-W_{-k, \mu}\left(-2 i \bar{\tau}^{1 / 2} \rho_{2}^{*}\right)\right. \\
& \times\left\{\frac{\left(1+2 \mu-4 i \widehat{\tau}^{1 / 2} \rho_{2}^{*}\right)}{2 i \widehat{\tau}^{1 / 2} \rho_{2}^{*}} W_{k, \mu}\left(2 i \widehat{\tau}^{1 / 2} \rho_{2}^{*}\right)\right. \\
& \left.\left.+\frac{(2 k-2 \mu-1)}{\left(2 i \hat{\tau}^{1 / 2} \rho_{2}^{*}\right)^{1 / 2}} W_{k-1 / 2, \mu+1 / 2}\left(2 i \widehat{\tau}^{1 / 2} \rho_{2}^{*}\right)\right\}\right) .
\end{aligned}
$$

By using (44) to (48) we obtain

$$
\begin{aligned}
& \Psi_{-}^{-}(r, \widehat{\tau}) \equiv \Theta(r, \widehat{\tau})+n^{+}(\widehat{\tau}) \Phi(r, \widehat{\tau}) \in L^{2}\left(\left[a, \rho_{2}^{*}\right]\right) \\
& \Psi_{-}^{+}(r, \widehat{\tau}) \equiv \Theta(r, \widehat{\tau})+n^{-}(\widehat{\tau}) \Phi(r, \widehat{\tau}) \in L^{2}\left(\left[\rho_{2}^{*}, 0\right]\right),
\end{aligned}
$$

where

$$
n^{+}(\widehat{\tau})=-\lim _{r \rightarrow 0} \frac{\Theta(r, \widehat{\tau})}{\Phi(r, \widehat{\tau})}, \quad n^{-}(\widehat{\tau})=-\lim _{r \rightarrow a} \frac{\Theta(r, \widehat{\tau})}{\Phi(r, \widehat{\tau})} .
$$

Therefore, the eigenfunctions for $I_{1} \cup I_{2}$ are

$$
\Psi_{-}(r, \widehat{\tau})= \begin{cases}\Psi_{-}^{-}(r, \widehat{\tau}), & r \in\left[a, \rho_{2}^{*}\right] \\ \Psi_{-}^{+}(r, \widehat{\tau}), & r \in\left[\rho_{2}^{*}, 0\right],\end{cases}
$$

and these automatically satisfy the boundary conditions at $a$. From (43) and (51) we find that

$$
\lim _{r \rightarrow 0^{-}} \Psi_{-}(r, \widehat{\tau})=\lim _{r \rightarrow 0^{+}} \Psi_{+}(r, \widehat{\tau})=0 .
$$

We then conclude that the eigenfunctions solutions to (5) with the accompanying boundary conditions would be

$$
\Psi(r, \widehat{\tau})= \begin{cases}\Psi_{+}(r, \widehat{\tau}), & r \in[a, 0] \\ \Psi_{-}(r, \widehat{\tau}), & r \in[0, b] .\end{cases}
$$

This completes the proof of the theorem.

\section{Computation of the Eigenvalues}

As in [11], the eigenvalues for our problem are $\widehat{\tau}^{(n)}$. To derive the eigenvalue relations for boundary value problems for which the eigenfunctions are obtained using a split interval technique requires a careful examination of the behaviour of the eigenfunctions. To enable us achieve our goal for the interval $r \in[a, b], a<0<b$, we will need the following conditions: 
(i) that the eigenfunctions solutions are valid for

$$
\arg \left(2 i \widehat{\tau}^{1 / 2} r k\right)<2 \pi,
$$

(ii) that

$$
\begin{aligned}
& \sin \left[2 \sqrt{2 i \widehat{\tau}^{1 / 2} b k}-\pi\left(\mu+\frac{1}{4}\right)\right] \\
& =\cos \left[2 e^{ \pm(1 / 2) \pi i} 2 \sqrt{2 i \widehat{\tau}^{1 / 2} b k}-\pi\left(\mu+\frac{1}{4}\right)\right],
\end{aligned}
$$

(iii) and that

$$
\begin{aligned}
& \sin \left[2 \sqrt{2 i \hat{\tau}^{1 / 2} a k}-\pi\left(\mu+\frac{1}{4}\right)\right] \\
& =\cos \left[2 e^{ \pm(1 / 2) \pi i} 2 \sqrt{2 i \widehat{\tau}^{1 / 2} a k}-\pi\left(\mu+\frac{1}{4}\right)\right] .
\end{aligned}
$$

We summarise our results in this section in the following theorem.

Theorem 2. The nonstandard SL eigenproblem (or the Schrödinger radial operator in $\mathbb{R}),(5)$ with (6) with separated turning points, which have solutions (53) and satisfy conditions (54)-(56) have its eigenvalues given by

$$
\widehat{\tau}^{(n)}=\frac{n^{4} \pi^{4}}{[8 i(b+a)+16 k \sqrt{a b}]^{2}} .
$$

Proof. The asymptotic expansions of the eigenfunctions that meet the requirement (54) are

$$
\begin{aligned}
& \frac{M_{k, \mu}\left(2 i \hat{\tau}^{1 / 2} r\right)}{\Gamma(1+2 \mu)} \\
& \sim\left(2 i \hat{\tau}^{1 / 2} r\right)^{1 / 4} \pi^{-1 / 2} k^{-1 / 4-\mu} \cos \left[2 \sqrt{2 i \hat{\tau}^{1 / 2} r k}-\pi\left(\mu+\frac{1}{4}\right)\right] \\
& \quad \times\left\{1+O\left(|k|^{-1 / 2}\right)\right\} \\
& \frac{M_{-k, \mu}\left(2 i \hat{\tau}^{1 / 2} r\right)}{\Gamma(1+2 \mu)} \\
& \sim\left(2 i \hat{\tau}^{1 / 2} r\right)^{1 / 4} \pi^{-1 / 2} k^{-1 / 4-\mu} \\
& \quad \times e^{\mp \pi i(\mu+1 / 4)} \cos \left[2 e^{ \pm(1 / 2) \pi i} \sqrt{2 i \hat{\tau}^{1 / 2} r k}-\pi\left(\mu+\frac{1}{4}\right)\right] \\
& \quad \cdot\left\{1+O\left(|k|^{-1 / 2}\right)\right\}
\end{aligned}
$$

where as usual $k=i z \widehat{\tau}^{-1 / 2}$ and $\mu=l+1 / 2$.
We then express the asymptotic approximation of the function $\Theta(r, \widehat{\tau})$ as follows:

$$
\begin{aligned}
\Theta & (r, \widehat{\tau}) \\
= & \alpha_{0}\left(2 i \widehat{\tau}^{1 / 2} r\right)^{1 / 4} \pi^{-1 / 2} \\
& \times k^{-1 / 4-\mu} \cos \left[2 \sqrt{2 i \widehat{\tau}^{1 / 2} r k}-\pi\left(\mu+\frac{1}{4}\right)\right] \\
& \cdot\left\{1+O\left(|k|^{-1 / 2}\right)\right\} \\
& +\alpha_{1}\left(2 i \bar{\tau}^{1 / 2} r\right)^{1 / 4} \pi^{-1 / 2} k^{-1 / 4-\mu} \\
& \times e^{\mp \pi i(\mu+1 / 4)} \cos \left[2 e^{ \pm(1 / 2) \pi i} \sqrt{2 i \hat{\tau}^{1 / 2} r k}-\pi\left(\mu+\frac{1}{4}\right)\right] \\
& \cdot\left\{1+O\left(|k|^{-1 / 2}\right)\right\} .
\end{aligned}
$$

Through a careful limiting process we find that

$$
\lim _{r \rightarrow a} n^{+}(\widehat{\tau}) \Phi(r, \widehat{\tau})=\lim _{r \rightarrow b} m^{+}(\widehat{\tau}) \Phi(r, \widehat{\tau})=0,
$$

a result that comes in handy.

Using (60) and (59), we apply the boundary conditions at $a$ and $b$ to obtain

$$
\begin{aligned}
& \alpha_{0} \cos \left[2 \sqrt{2 i \widehat{\tau}^{1 / 2} a k}-\pi\left(\mu+\frac{1}{4}\right)\right] \\
& +\alpha_{1} e^{\mp \pi i(\mu+1 / 4)} \cos \left[2 e^{ \pm(1 / 2) \pi i} \sqrt{2 i \hat{\tau}^{1 / 2} a k}-\pi\left(\mu+\frac{1}{4}\right)\right] \\
& =0, \\
& \alpha_{0} \cos \left[2 \sqrt{2 i \widehat{\tau}^{1 / 2} b k}-\pi\left(\mu+\frac{1}{4}\right)\right] \\
& \quad+\alpha_{1} e^{\mp \pi i(\mu+1 / 4)} \cos \left[2 e^{ \pm(1 / 2) \pi i} \sqrt{2 i \hat{\tau}^{1 / 2} b k}-\pi\left(\mu+\frac{1}{4}\right)\right] \\
& =0 .
\end{aligned}
$$

By employing the conditions in (55) and (56), we obtain from (61) that

$$
\sin \left[2 \sqrt{2 i \widehat{\tau}^{1 / 2} b k}-2 \sqrt{2 i \widehat{\tau}^{1 / 2} a k}\right]=0 .
$$

Thus, the eigenvalues $\widehat{\tau}^{(n)}$ may be expressed as

$$
\widehat{\tau}^{(n)}=\frac{n^{4} \pi^{4}}{[8 i(b+a)+16 k \sqrt{a b}]^{2}},
$$

where $n$ is the mode number. The eigenvalues are complex, which is consistent with the fact that $\tau$ in the radial Schrödinger equation is complex. This result as expected is similar to the result obtained in [11] since the perturbation term $\varepsilon$ which is an integral part of $\widehat{\tau}$ is independent of $r$. 


\section{Concluding Remarks}

The boundary value problem with interior singularities and turning points for which resonance equations result may be represented as nonstandard Sturm-Liouville eigenproblems. With the asymptotic expansions of the eigenfunctions solutions for $r \in \mathbb{R}^{3}$ being the same for $r \in \mathbb{R}$, we have in this paper presented solutions valid for an unrestricted interval. The three turning points analysis presented here when the perturbation term $\varepsilon$ is a function of $r$ demonstrate the applicability of the method in these cases. The beauty of this split interval technique which employs the use of the Titchmarsh-Weyl $m$-function theory is that eigenfunctions solutions could also be obtained for the nonconstant perturbation term $q(r)=\varepsilon_{0}+\varepsilon_{1} r$ which happens to have three turning points provided one of these turning points coalesce with another, thus permitting us to use the branch cuts as in the theory of atmospheric and oceanic waves.

\section{Conflict of Interests}

The author declares that there is no conflict of interests regarding the publication of this paper.

\section{References}

[1] D. Eric, Exact WKB Analysis near a Simple Turning Point. Algebraic Analysis of Differential Equations from Micro-Local Analysis to Exponential Asymtotics, Springer, Tokyo, Japan, 2008.

[2] A. Fruchard and R. Schäfke, "Composite asymptotic expansions and turning points of singularly perturbed ordinary differential equations," Comptes Rendus Mathematique, vol. 348, no. 23-24, pp. 1273-1277, 2010.

[3] H.-R. Sun, Y.-N. Li, J. J. Nieto, and Q. Tang, "Existence of solutions for Sturm-Liouville boundary value problem of impulsive differential equations," Abstract and Applied Analysis, vol. 2012, Article ID 707163, 19 pages, 2012.

[4] L. Zhang, X. Huang, and C. Xing, "Solvability of SturmLiouville boundary value problems with impulses," Boundary Value Problems, vol. 2013, article 192, 2013.

[5] M. Jiaqi, "Singularly perturbed solution of boundary value problem for nonlinear equations of fourth order with two parameters," Advances in Mathematics, vol. 39, no. 6, pp. 736740, 2010.

[6] M. Ni and Z. Wang, "On step-like contrast structure of singularly perturbed systems," Boundary Value Problems, vol. 2009, Article ID 634324, 17 pages, 2009.

[7] Y. Tian and W. Ge, "Multiple positive solutions for a second order Sturm-Liouville boundary value problem with a $p$ Laplacian via variational methods," The Rocky Mountain Journal of Mathematics, vol. 39, no. 1, pp. 325-342, 2009.

[8] Y. Tian and W. Ge, "Multiple solutions of impulsive SturmLiouville boundary value problem via lower and upper solutions and variational methods," Journal of Mathematical Analysis and Applications, vol. 387, no. 2, pp. 475-489, 2012.

[9] Y. Tian and W. Ge, "Variational methods to Sturm-Liouville boundary value problem for impulsive differential equations," Nonlinear Analysis: Theory, Methods \& Applications, vol. 72, no. 1, pp. 277-287, 2010.
[10] T. M. Acho and D. P. Clemence, "Sturm-Liouville eigenproblems with an interior double pole," Zeitschrift für Angewandte Mathematik und Physik, vol. 46, no. 3, pp. 459-474, 1995.

[11] T. M. Acho, "Derivation of eigenrelations for the SturmLiouville boundary value problems with interior singularities," Quarterly of Applied Mathematics, vol. 65, no. 2, pp. 375-383, 2007.

[12] J. P. Boyd, "Sturm-Liouville eigenproblems with an interior pole," Journal of Mathematical Physics, vol. 22, no. 8, pp. 15751590, 1981.

[13] F. V. Atkinson, N. N. Everitt, and A. Zettl, "Regularisation of a Sturm-Liouville problem with an interior singularity," Differential Integral Equations, vol. 1, no. 2, pp. 213-221, 1988.

[14] J. P. Boyd, "Complex coordinate methods for hydrodynamic instabilities and Sturm-Liouville eigenproblems with an interior singularity," Journal of Computational Physics, vol. 57, no. 3, pp. 454-471, 1985.

[15] T. M. Acho, "A matching technique and structure theorems for Sturm-Liouville boundary value problems with interior singularities," Quaestiones Mathematicae, vol. 25, no. 3, pp. 275287, 2002.

[16] T. M. Acho and D. P. Clemence, "The parameter dependent Sturm-Liouville eigenproblem with an interior simple or double pole," The ANZIAM Journal, vol. 43, no. 4, pp. 479-491, 2002.

[17] E. A. Coddington and N. Levinson, Theory of Ordinary Differential Equations, R.E. Krieger, Malabar, Fla, USA, 1955.

[18] Y. Pinchover and J. Rubinstein, An Introduction to Partial Differential Equations, Cambridge University Press, Cambridge, UK, 2007.

[19] M. A. Shubov, "High-energy asymptotics of resonances for three-dimensional Schrödinger operator with screened Coulomb potential," Journal of Mathematical Physics, vol. 35, no. 2, pp. 656-679, 1994.

[20] R. G. Newton, Scattering Theory of Waves and Particles, Springer, New York, NY, USA, 2nd edition, 1982.

[21] L. J. Slater, Confluent Hypergeometric Functions, Cambridge University Press, 1960.

[22] N. Bleistein and R. A. Handelsman, Asymptotic Expansion of Integrals, Dover Publications, Ney York, NY, USA, 1986.

[23] G. F. Carrier, M. Krook, and C. E. Pearson, Functions of a Complex Variable, vol. 49 of Classics in Applied Mathematics, SIAM, Philadelphia, Pa, USA, 2005.

[24] A. H. Nayfeh, Perturbation Methods, John Wiley \& Sons, New York, NY, USA, 1973.

[25] M. Abramowitz and I. Stegun, Handbook of Mathematical Functions, Dover, New York, NY, USA, 1965. 


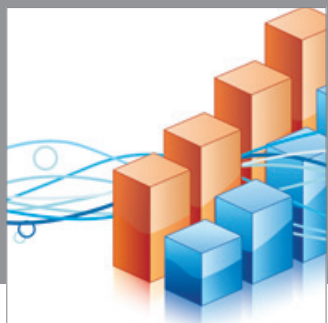

Advances in

Operations Research

mansans

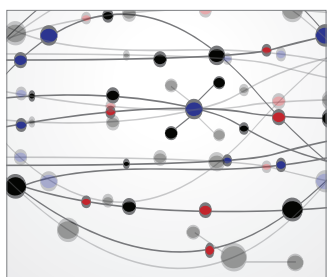

The Scientific World Journal
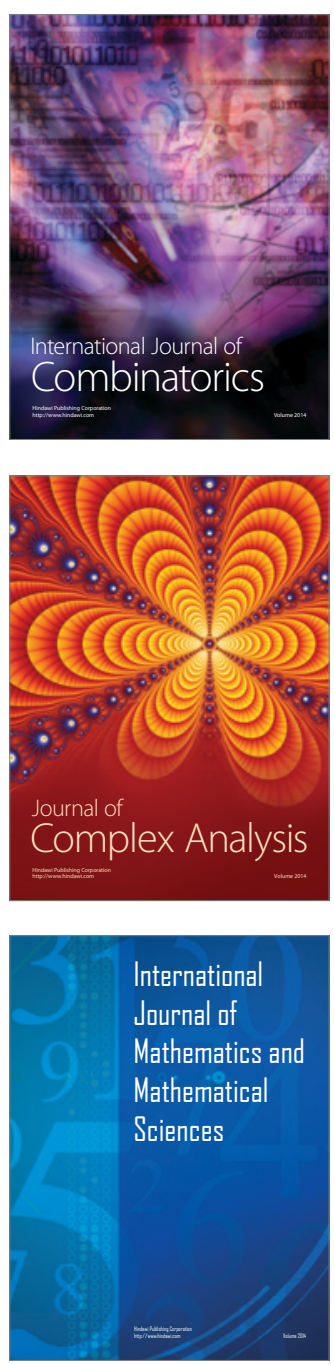
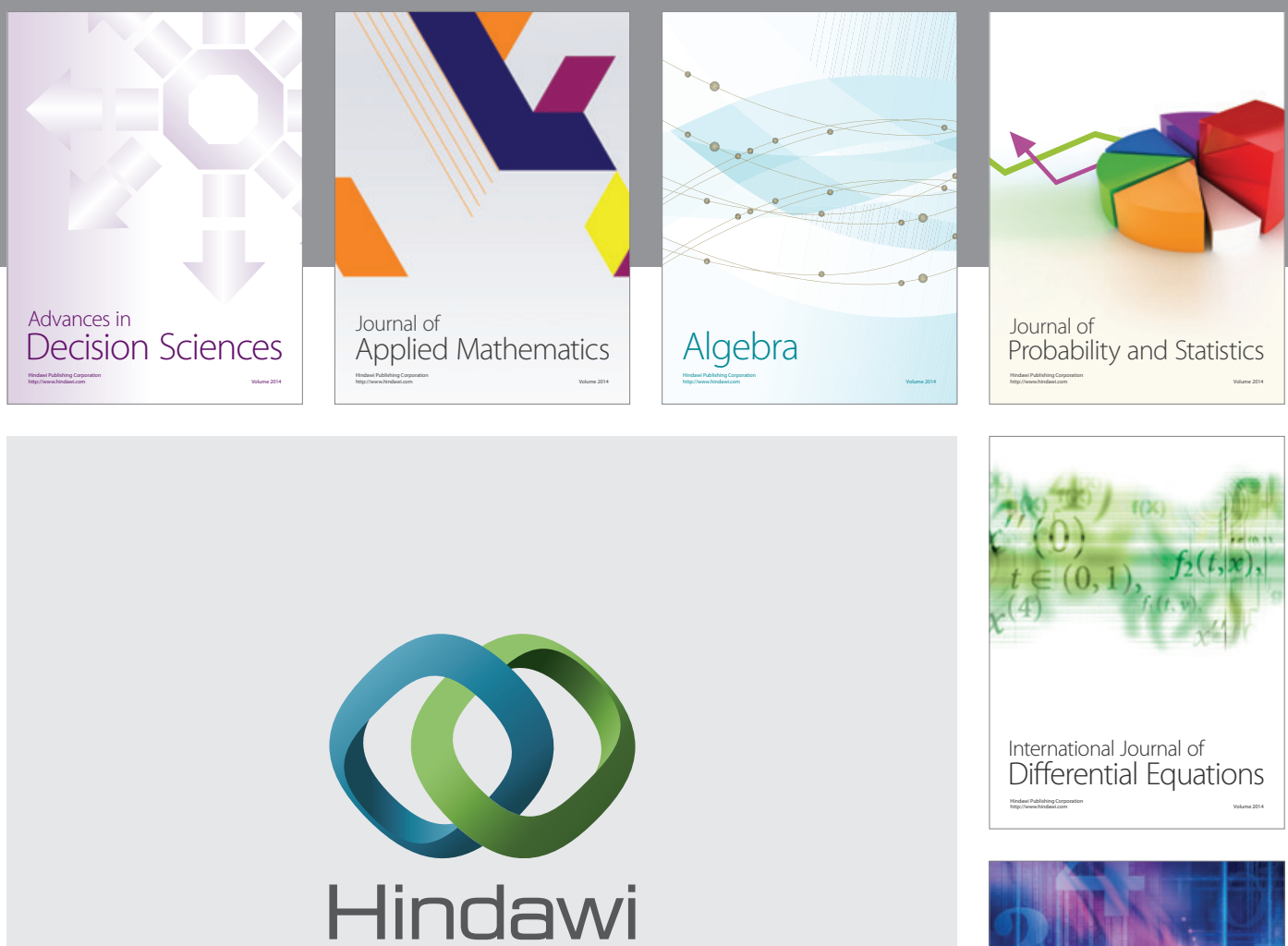

Submit your manuscripts at http://www.hindawi.com
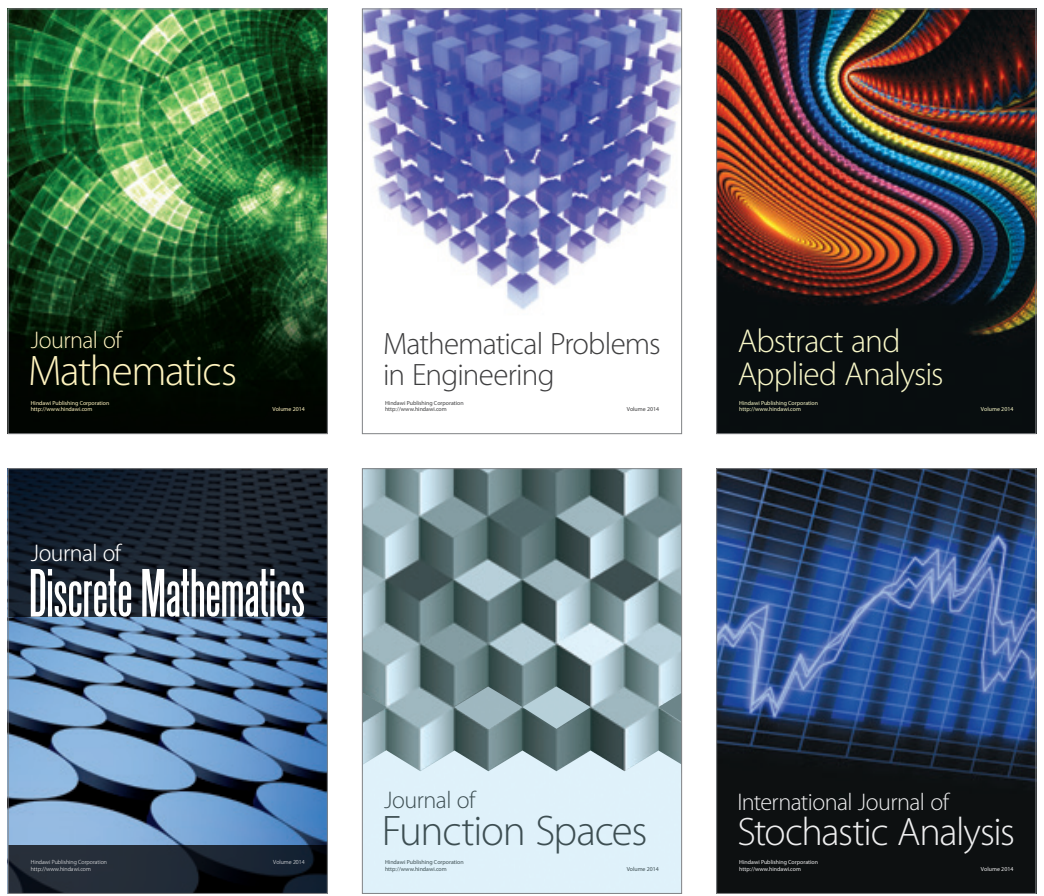

Journal of

Function Spaces

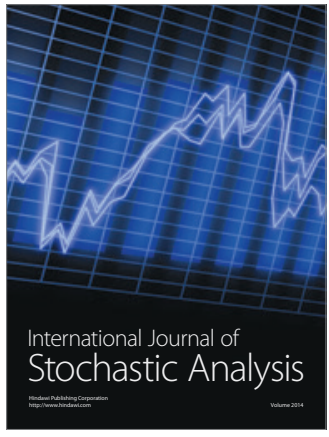

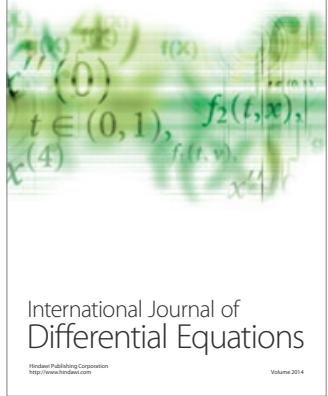
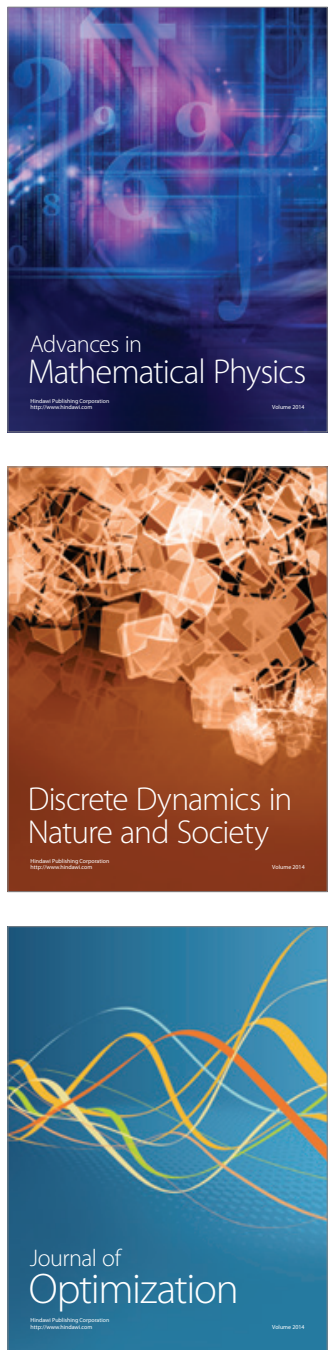